\title{
Letter to the Editor: Notice of Errors in Three Previous Papers Reporting Measures of Hyoid and Laryngeal Position
}

\author{
Catriona M. Steele ${ }^{1,2}$ D $\cdot$ Melanie Peladeau-Pigeon ${ }^{1}$
}

Received: 26 June 2020 / Accepted: 27 July 2020 / Published online: 1 August 2020

(c) Springer Science+Business Media, LLC, part of Springer Nature 2020

To the Editor,

We are writing to inform the readers of the Dysphagia journal of errors in the results of three papers, all published in 2019. All three of these papers reported measures either of hyoid bone or laryngeal position from videofluoroscopy recordings of swallows, calculated using a downloadable spreadsheet that had been made available as an open source resource on the Swallowing Rehabilitation Research Lab (SRRL) website https://www.steeleswallowinglab.ca. It has recently come to our attention that the mathematical formulas that were embedded in that peak position calculation spreadsheet contained an error, such that the output for peak $\mathrm{X}$ (anterior) and peak Y (superior) position was reversed. None of the other measures generated in the spreadsheet were impacted by the error. Please find below a summary of the actions taken to address this error:

(1) Removal of the Spreadsheet Containing the Error

The spreadsheet containing the error did not have a date or version code but has been removed from the SRRL as of June, 2019. A corrected spreadsheet (with a version code) can be obtained by reaching out to the SRRL directly at TRISwallowingLab@uhn.ca.

(2) Support for Researchers who may have used the Spreadsheet Containing the Error

To our knowledge, there are three manuscripts in the Dysphagia journal that contain erroneous measures for peak anterior and peak superior hyoid position measures, based on use of the spreadsheet containing the error [1-3]. Additionally, any researchers who are concerned that they may have used the spreadsheet containing the error are encouraged to contact the SRRL by email at TRI-SwallowingLab@ uhn.ca for assistance in confirming whether their data have been impacted.

\section{References}

1. Brates D, Molfenter SM, Thibeault SL. Assessing hyolaryngeal excursion: comparing quantitative methods to palpation at the bedside and visualization during video fluoroscopy. Dysphagia. 2019;34(3):298-307.

2. Molfenter SM, Lenell C, Lazarus CL. Volumetric changes to the pharynx in healthy aging: consequence for pharyngeal swallow mechanics and function. Dysphagia. 2019;34(1):129-37.

3. Riley A, Miles A, Steele CM. An exploratory study of hyoid visibility, position, and swallowing-related displacement in a pediatric population. Dysphagia. 2019;34(2):248-56.

Publisher's Note Springer Nature remains neutral with regard to jurisdictional claims in published maps and institutional affiliations.

Catriona M. Steele $\mathrm{PhD}$

Melanie Peladeau-Pigeon MHSc
Catriona M. Steele

catriona.steele@uhn.ca

1 The KITE Research Institute - Toronto Rehabilitation Institute, University Health Network, Toronto, Canada

2 Faculty of Medicine, Rehabilitation Sciences Institute, University of Toronto, Toronto, Canada 